\title{
Is the Grand Tack model compatible with the orbital distribution of main belt asteroids?
}

\author{
Rogerio Deienno ${ }^{\mathrm{a}}$, Rodney S. Gomes ${ }^{\mathrm{b}}$, Kevin J. Walsh ${ }^{\mathrm{c}}$, Alessandro Morbidellid ${ }^{\mathrm{d}}$, David \\ Nesvornýc \\ ${ }^{a}$ Instituto Nacional de Pesquisas Espaciais, Avenida dos Astronautas 1758, CEP 12227-010 São José dos Campos, SP, \\ Brazil \\ ${ }^{b}$ Observatório Nacional, Rua General José Cristino 77, CEP 20921-400 Rio de Janeiro, RJ, Brazil \\ ${ }^{c}$ Department of Space Studies, Southwest Space Research Institute, 1050 Walnut St., Boulder, CO 80302, USA \\ ${ }^{d}$ Laboratoire Lagrange, UMR7293, Université Côte d'Azur, CNRS, Observatoire de la Côte d'Azur, Boulevard de \\ l'Observatoire, 06304 Nice Cedex 4, France
}

\begin{abstract}
The Asteroid Belt is characterized by the radial mixing of bodies with different physical properties, a very low mass compared to Minimum Mass Solar Nebula expectations and has an excited orbital distribution, with eccentricities and inclinations covering the entire range of values allowed by the constraints of dynamical stability. Models of the evolution of the Asteroid Belt show that the origin of its structure is strongly linked to the process of terrestrial planet formation. The Grand Tack model presents a possible solution to the conundrum of reconciling the small mass of Mars with the properties of the Asteroid Belt, including the mass depletion, radial mixing and orbital excitation. However, while the inclination distribution produced in the Grand Tack model is in good agreement with the one observed, the eccentricity distribution is skewed towards values larger than those found today. Here, we evaluate the evolution of the orbital properties of the Asteroid Belt from the end of the Grand Tack model (at the end of the gas nebula phase when planets emerge from the dispersing gas disk), throughout the subsequent evolution of the Solar System including an instability of the Giant Planets approximately 400 My later. Before the instability, the terrestrial planets were modeled on dynamically cold orbits with Jupiter and Saturn locked in a 3:2 mean motion resonance. The model continues for an additional 4.1 Gy after the giant planet instability. Our results show that the eccentricity distribution obtained in the Grand Tack model evolves towards one very similar to that currently observed, and the semimajor axis distribution does the same. The inclination distribution remains nearly unchanged with a slight preference for depletion at low inclination; this leads to the conclusion that the inclination distribution at the end of the Grand Tack is a bit over-excited. Also, we constrain the primordial eccentricities of Jupiter and Saturn, which have a major influence on the dynamical evolution of the Asteroid Belt and its final orbital structure.
\end{abstract}

Keywords: Asteroids, dynamics, Origin, Solar System, Planetary dynamics, Planets, migration

Email address: rogerio.deienno@gmail.com (Rogerio Deienno) 


\section{Introduction}

The Asteroid Belt is challenging to understand but is critical for studies of the formation and early evolution of the Solar System. The orbital configuration of the Asteroid Belt is believed to have been established in two phases. The first phase dates back to the first few million years of Solar System's formation and should be studied in conjunction with the formation of the inner and outer planets, especially Jupiter and Saturn. The second phase occurred when the Asteroid Belt witnessed a Giant Planet instability, long after the damping effects of the gaseous Solar Nebula had dissipated

In general, simulations of the dynamical re-shaping of the Asteroid Belt are made in conjunction with the formation of the inner planets. The first simulations of terrestrial planet formation (Chambers and Wetherill, 1998) included a set of planetary embryos uniformly distributed in the inner region of the Solar System with orbits initially dynamically cold (low eccentricity and inclination). Through numerical integrations of the equations of motion of these embryos, adding a model of accretion by collisions, the system evolves to form planets in the inner region of the Solar System on stable orbits. While early results about the formation of terrestrial planets were promising, one of the problems found in these integrations was related with the final eccentricities of the planets, which were systematically larger than the real ones. The models produced more promising results when the presence of a substantial population of planetesimals was also accounted for; in fact, the dynamical friction exerted by the planetesimals acted to decrease the excitation of the planet's final orbits (Chambers, 2001; O'Brien et al., 2006).

An important ingredient was the presence of Jupiter, which should have completed its formation much earlier than the inner planets (Chambers and Wetherill, 1998; Chambers, 2001; Petit et al., 2001). Primarily, the influence of Jupiter on the Asteroid Belt is to promote destructive collisions (fragmentation) rather than constructive collisions (accretion) (Petit et al., 2002). However, Jupiter alone can not excite the eccentricity of planetesimals so much as to explain the current excited orbits of asteroids (Petit et al., 2002). In addition, there is significant diversity in the physical properties of asteroids found in the Main Asteroid Belt, but their main taxonomic classes are found in roughly overlapping distributions - although S-class bodies predominate in the inner regions and C-class bodies in the outer regions (see DeMeo and Carry, 2014). The solution of these issues have been attributed to the original presence of planetary embryos in the Asteroid Belt (Petit et al., 1999; O'Brien et al., 2007). These embryos, once excited by Jupiter, would have scattered the orbits of the planetesimals. In the end, the Asteroid Belt would have been depleted of planetesimals and totally devoid of embryos.

Despite the many successes in the modeling of the terrestrial planets and Asteroid Belt by the simulations described above, systematic problems persisted. The planet formed in the approximate region of Mars systematically showed a much larger mass than the real Mars (see Raymond et al., 2009). An experiment by Hansen (2009) found that if there is sharp outer edge in the initial mass distribution of solids at about $1.0 \mathrm{AU}$, then the models consistently reproduce the mass of Mars.

Walsh et al. (2011) proposed a mechanism to modify the original mass distribution of solids and produce the truncated disk explored by Hansen (2009), by accounting for the early migration of Jupiter and Saturn when they were still embedded in the gaseous proto-planetary disk. An outcome found in many hydrodynamical models (Masset and Snellgrove, 2001; Morbidelli et al., 2007; Pierens and Nelson, 2008; Pierens and Raymond, 2011; D'Angelo and Marzari, 2012) of the interaction between giant planets and gaseous disks is that the type-II inward migration of a Jupiter-mass planet is halted and even reversed when a second, less massive planet, is formed 
external to the first one. This provides the explanation for why Jupiter did not migrate to very close the Sun, as is seen for giant planets in many other planetary systems (Udry and Santos, 2007; Cumming et al., 2008). Instead, Jupiter would have migrated first inwards, then outwards. Because of the change in direction of the orbital motion of Jupiter (a "tack" in sailor's jargon), the Walsh et al. (2011) model is named the "Grand Tack". The timing of the formation of Saturn is constrained by the mass distribution of the terrestrial planets, which are best reproduced when Jupiter reverses migration at $1.5 \mathrm{AU}$ and truncates the disk at $1 \mathrm{AU}$.

The migration of Jupiter would have strongly affected any planetesimals formed in the presentday Asteroid Belt, with a primary consequence of substantially depleting the entire region of small bodies. The inward migration phase primarily pushes the asteroids originally inside of Jupiter's orbit (named "S-class" in Walsh et al. (2011)) down to lower semimajor axes (inside of $1 \mathrm{AU}$ ), though Walsh et al. (2011) found that about $10 \%$ of these bodies are scattered outward onto orbits with semimajor axis $a$ between 4-10 AU. During the outward migration of Jupiter and Saturn, these bodies are encountered again, and about $1 \%$ are scattered back into the Asteroid Belt. Meanwhile Jupiter and Saturn eventually encounter primitive planetesimals (titled "C-class" in Walsh et al. (2011)), and a fraction of a percent of these are also scattered into the Asteroid Belt. This provides, at the time when the gas nebula has dispersed, a final belt which is depleted in mass by a factor of about 1,000 , that contains two different classes of bodies partially mixed in heliocentric distance and with orbits excited in eccentricities and inclinations (although the final eccentricity distribution does not match well the current one, as discussed below).

Numerous constraints, such as the ages of the last impact basins on the Moon (Bottke et al., 2007), the impact age distribution of HED meteorites (Marchi et al., 2013), and the small total chondritic mass accreted by the Moon since its formation (Morbidelli et al., 2012), point to an epoch of increased bombardment in the inner Solar System about $~ 400-700$ My after the removal of gas from the proto-planetary disk (whereas the Grand Tack happened before the removal of the gas). This period of increased bombardment is usually called "Terminal Lunar Cataclysm" or "Late Heavy Bombardment" (LHB) (see Hartmann et al., 2000; Chapman et al., 2007, for reviews), and we will adopt the LHB nomenclature here. The origin of the LHB has been linked to a dynamical upheaval in the outer Solar System frequently referred to as the "Nice model" (Gomes et al., 2005; Levison et al., 2011; Bottke et al., 2012). During this dynamical upheaval the giant planets would have suffered an instability and a period of mutual close encounters that radically changed their orbits. In turn, the orbital change of the giant planets would have severely affected the distribution of the asteroids in the main belt (Morbidelli et al., 2010). The best guess on when this instability occurred, from various constraints, is 4.1 Gy ago (Bottke et al., 2012; Morbidelli et al., 2012).

This is important because the final Asteroid Belt in Walsh et al. (2011) lacks objects with small eccentricities. Indeed, according to the Grand Tack model, the eccentricity distribution expected for the Asteroid Belt at the time when the gas nebula dispersed, some 3-10 Myr after the emergence of first solids and roughly $4.5 \mathrm{Gyr}$ ago, peaks around 0.4. On the other hand, the current distribution of the Asteroid Belt peaks around 0.1 (Morbidelli et al., 2015). It has never been studied whether the Grand Tack final distribution could evolve to one similar to what we see today due to the perturbations caused by the giant planet instability during the Nice Model. The goal of this paper is to present such a study.

In this work, we will study in detail the evolution of the Asteroid Belt orbital structure, from the end of the Grand Tack model to the giant planet orbital instability, through the instability phase, and finally during the last $\sim 4$ Gy until today.

This work will therefore unfold as follows: section 2 explains our Solar System's configura- 

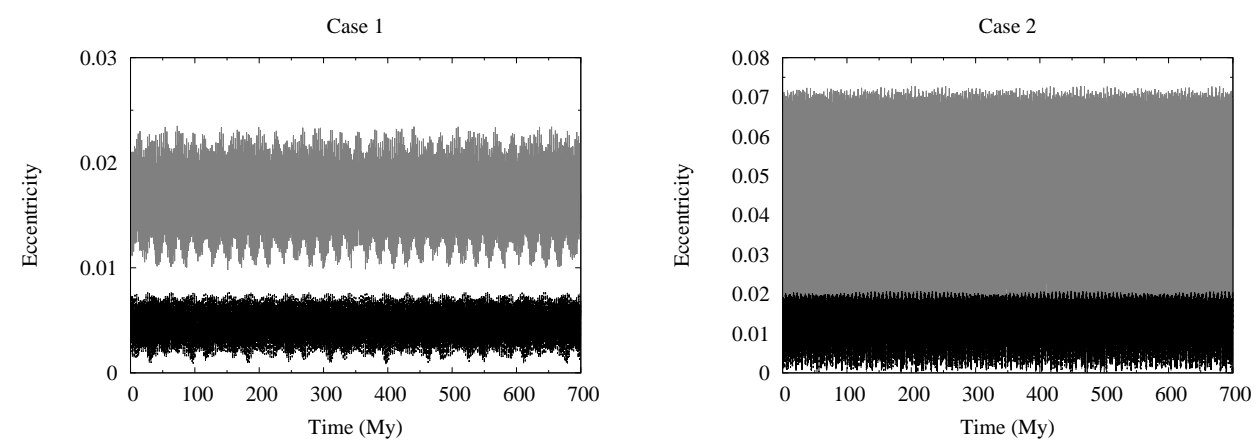

Figure 1: Eccentricity behavior of Jupiter (black) and Saturn (gray) before the planetary instability as a function of time. These two planets are in coplanar orbits, and locked in their 3:2 MMR with Jupiter at 5.4 AU. Left: Case 1 is a initial configuration from Morbidelli et al. (2007) where planets evolve into a resonant configuration in a hydrodynamical simulation. Right: Case 2 is an arbitrary case where planets are placed in resonant orbits, allowing large variations in eccentricity.

tion and the method used for the numerical simulations. In section 3 we discuss the dynamical evolution of the Asteroid Belt throughout the various phases of the Solar System's evolution, as well as the influence of the primordial eccentricities of Jupiter and Saturn on the final structure of the Asteroid Belt. Finally, section 4 summarizes the main conclusions of this paper.

A complementary study, approaching the problem from a different perspective, has been recently presented in Roig and Nesvorný (2015). We will compare our results with theirs at the end of section 3.2

\section{Methods}

Our numerical simulations contain five planets, Venus, Earth, Mars, Jupiter, and Saturn (all with their current masses), plus 10,000 massless particles representing the final outcome of the Asteroid Belt in the Grand Tack simulations (described below). Uranus, Neptune, and the putative extra ice giant invoked in Nesvorný and Morbidelli (2012) are not included in any of our simulations (the same applies for the planet Mercury). These planets are indeed too far from the Asteroid Belt to have any important direct effect on its structure. A caveat, however, is that in some simulations the extra ice giant is sent temporarily onto an Asteroid Belt-crossing orbit before being ultimately ejected from the Solar System. In the case the Asteroid Belt-crossing phase last for sufficiently long time, the effects of this body on the Asteroid Belt should be taken into account (see Brasil et al., 2016). Nevertheless, because we have limited understanding on how exactly the evolution of the planets occurred (i.e. how deep a planet crossed the Asteroid Belt and for how long), we prefer to neglect this putative Asteroid Belt-crossing event and focus only on the effects induced by Jupiter, Saturn and the terrestrial planets, whose evolution is better constrained.

The orbital configuration of the five planets was chosen as follows:

- Before the planetary instability: The terrestrial planets are modeled to be on simple planar orbits (Brasser et al., 2013). Jupiter and Saturn, also on planar orbits, were locked in their mutual 3:2 MMR with Jupiter at 5.4 AU. Two cases, differing for the eccentricity of the giant planets (Fig. 1) are considered. Case 1 (left) is an initial configuration from the 
hydrodynamical simulations of Morbidelli et al. (2007), where the planets migrated into the resonance and preserved orbits with very small eccentricities due to the tidal damping effect exerted by the gas disk. Case 2 (right) is an arbitrary case where the planets are placed in resonant orbits, but with larger eccentricities (although still smaller than their current values). For this phase (for both cases), the numerical integrations are continued for $400 \mathrm{My}$ (i.e. until $4.1 \mathrm{~Gy} \mathrm{ago}$ ), the best estimate of the time of the giant planet instability (Gomes et al., 2005; Bottke et al., 2012; Morbidelli et al., 2012).

- After the planetary instability: The initial asteroid's orbits are those recorded at the end of the simulations of the previous phase, but the planets are placed onto their presentday orbits. By doing this, we model the instantaneous transition of the planets from their pre-instability orbits to the current orbits. In other words, we simulate the response of the Asteroid Belt to the change of the planetary orbits, but we assume that the actual dynamical path that the planets followed in this transition had no important effects. We take this simple approach because precisely what happened during the planetary instability is unknown, and many different paths could have been followed by the giant planets (Nesvorný and Morbidelli, 2012). Certainly, no instability simulation can have the pretension to reproduce exactly what happened. We know from previous studies (Brasser et al., 2009; Morbidelli et al., 2010) that the change of the giant planets' orbits had to be fast and of the "jumping-Jupiter" type, i.e. dominated by a few impulsive events due to mutual planetary encounters. Thus, we believe that substituting the original planetary orbits with the current orbits is the best approximation of the real planetary evolution we can implement without the risk of incurring into arbitrary simulation artefacts. In particular, by using the current planetary system, the locations and strengths of all resonances exactly match the real ones, whereas no singular instability simulation would finish with precisely a replica of the current-day planetary system. For the same reasons, this approach has already been adopted by Bottke et al. (2012) in their study of the projectile flux from the Asteroid Belt to the terrestrial planets. The opposite approach (i.e. simulating the dynamics of the giant planets during the instability phase) has been taken by Roig and Nesvorný (2015). The second phase of simulations were conducted for $4.1 \mathrm{~Gy}$, until a total timescale of $4.5 \mathrm{~Gy}$ was covered by the combination of the two phases of the Solar System evolution.

Concerning the asteroid distribution we consider two cases. In our nominal case we use the asteroid distribution resulting from the simplest of all Grand Tack scenarios, namely the one that only included Jupiter and Saturn (Figure 2. of Supplementary Information of Walsh et al. (2011), referenced as "2-planet Grand Tack simulation" hereafter). However, for comparison we also consider the distribution generated in the Grand Tack scenario that also include Uranus and Neptune (presented in the main paper of Walsh et al. (2011), referenced as "4-planet Grand Tack simulation" hereafter).

Because the original Grand Tack simulations had only $\sim 1,000$ particles in the final sample in the Asteroid Belt, in order to improve statistics we generated 10,000 massless particles matching the basic traits of the $a, e, i$ distribution obtained in the 2-planet Grand Tack simulation, with the following properties:

- Eccentricity distribution is a normal distribution with mean of 0.38 and sigma of 0.17 .

- Inclination distribution is a Rayleigh distribution with a sigma of 10 degrees. 
- Semimajor axis distribution is nearly an uniform distribution spread over the range 1.8-3.6 AU.

While these functional fits reproduce each distribution individually, combined they produced an excess of objects with high-eccentricity and low-inclination compared to the distribution of asteroids at the end of the Grand Tack simulations. To remove this excess, the 10,000 particles were re-sampled in bins of width 0.05 in eccentricity and 5 degrees in inclination. In each bin, we allowed the total number of particles to be at most 4 times more numerous than the asteroids at the end of the Grand Tack simulations in the same bin. If there were no original Grand Tack asteroids in the bin, a maximum of 4 particles from the functional fits was allowed. This resampling limited the excess population of high-eccentricity and low-inclination bodies found in the 10,000 particle distributions, and resulted in a final population of 6,424 bodies. The advantage of this procedure, relative to using directly the Grand Tack asteroids as initial conditions, is that the functional fits allow us to generate more particles (but this could have been achieved also by cloning the Grand Tack asteroids) and also place some particles in bins originally with zero Grand Tack asteroids, thus making the distribution smoother.

For the 4-planet Grand Tack simulation we simply resampled the above distribution attributing to each particle a "weight", representing the probability that said particle exists at the end of the 4-planet Grand Tack run. Because the semimajor axis and inclination distributions in the 2-planet and 4-planet Grand Tack simulations are basically the same, these weights are computed from the eccentricity distributions only, which are significantly different. These weights are then used to build a new final distribution from that obtained in our nominal case.

In all simulations all planets interact with each other while also perturbing the test particles. Test particles interact with the planets but not among themselves. The integrations have been conducted using Mercury (Chambers, 1999), in the Hybrid option with a time step of 10 days (more than 20 steps per orbital period of Venus).

\section{Results}

Figure 2 shows the current structure of the Asteroid Belt plotting all objects with absolute magnitude $\mathrm{H}<10$ from the Minor Planet Center catalog 1 , where the Main Asteroid Belt is assumed to be observationally complete at this size (Jedicke et al.,2002). Our goal is to verify if it is possible that the orbital configuration of the Asteroid Belt at the end of the Grand Tack model could evolve to one similar to that shown in this figure. To do so, we considered the two phases of the Solar System evolution previously discussed in section 2 .

\subsection{Before the planetary instability}

Figure 3 shows the current Asteroid Belt orbital distribution (gray line) compared with the initial conditions used in this experiment that were generated from the Grand Tack simulations (as described in section 2); the solid line is for the result of the 2-planet Grand Tack simulation and the dashed line is for the 4-planet simulation. With the exception of the inclination distributions, which match fairly well, the Grand Tack and the real distributions are clearly different, i.e., the eccentricity distributions in the Grand Tack simulations are skewed towards large values, and the semimajor axis distributions are nearly uniform instead of having the clear gaps found in today's

\footnotetext{
${ }^{1}$ http://www.minorplanetcenter.org/iau/MPCORB.html
} 


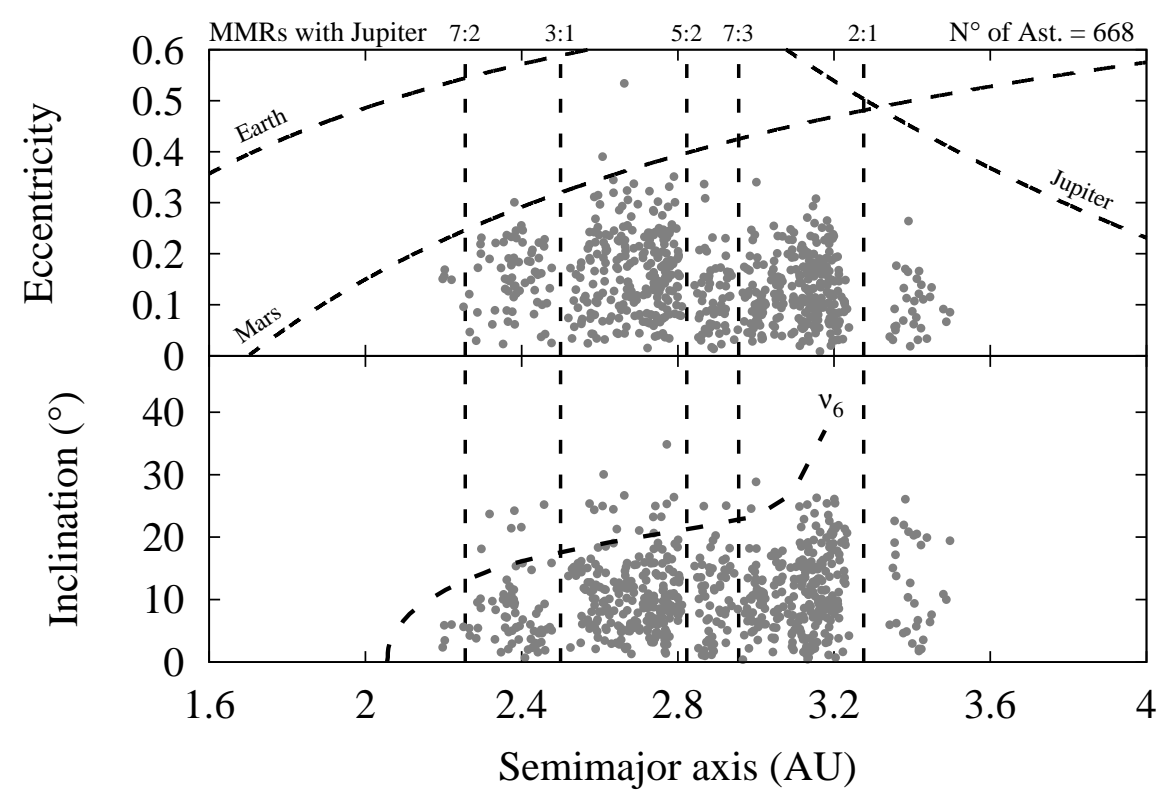

Figure 2: Current structure of the Asteroid Belt with all $\mathrm{H}<10$ objects from the Minor Planet Center catalog. Gray dots represent the asteroids. Curved dashed lines in the top panel represent the boundaries for Earth-, Mars-, and Jupitercrossing orbits (from left to right). In the bottom panel, the curved dashed line represents the current location of the $v_{6}$ secular resonance, which occurs when the precession rate of an asteroid's longitude of perihelion is equal to the mean precession rate of the perihelion of Saturn. Vertical dashed lines show MMRs between asteroids and Jupiter.

population. The 2-planet and 4-planet Grand Tack simulations also give eccentricity distributions quite different from each other, with the distribution obtained in the 4-planet simulation being even more skewed to large values. Many particles in the final Grand Tack distributions, however, must clearly be unstable, because their small semimajor axis and/or large eccentricity make them planet-crossing.

Figure 4, shows snapshots of the evolution of the Asteroid Belt in our simulations, starting from the final 2-planet Grand Tack configuration. The left panel illustrates the initial system, corresponding to the solid black lines of Fig. 3. Considering the evolution described in section 2 (with planets on pre-instability orbits), and using Case 1 (low-eccentricity giant planets orbits from Morbidelli et al. (2007)), after $400 \mathrm{My}$ of evolution we obtain the distribution depicted in the right panel of Figure 4. The colors of the particles in the left panels correspond to the particles' lifetimes in this simulation. Results related to Case 2 (giant planets with more eccentric orbits), will be considered only for measuring the influence of the primordial eccentricities of Jupiter and Saturn and presented in section 3.3 .

The distribution after $400 \mathrm{My}$ is already closer to the current one (see Fig. 2) because the very high eccentricity objects have been removed due to interactions with the planets whose orbits they crossed. The main difference with the current main belt distribution is that our simulated belt extends inwards of 2.2 AU in semimajor axis. This is because the powerful $v_{6}$ resonance is not in place yet, given that Jupiter and Saturn are still in resonance and on quasi-circular orbits. 

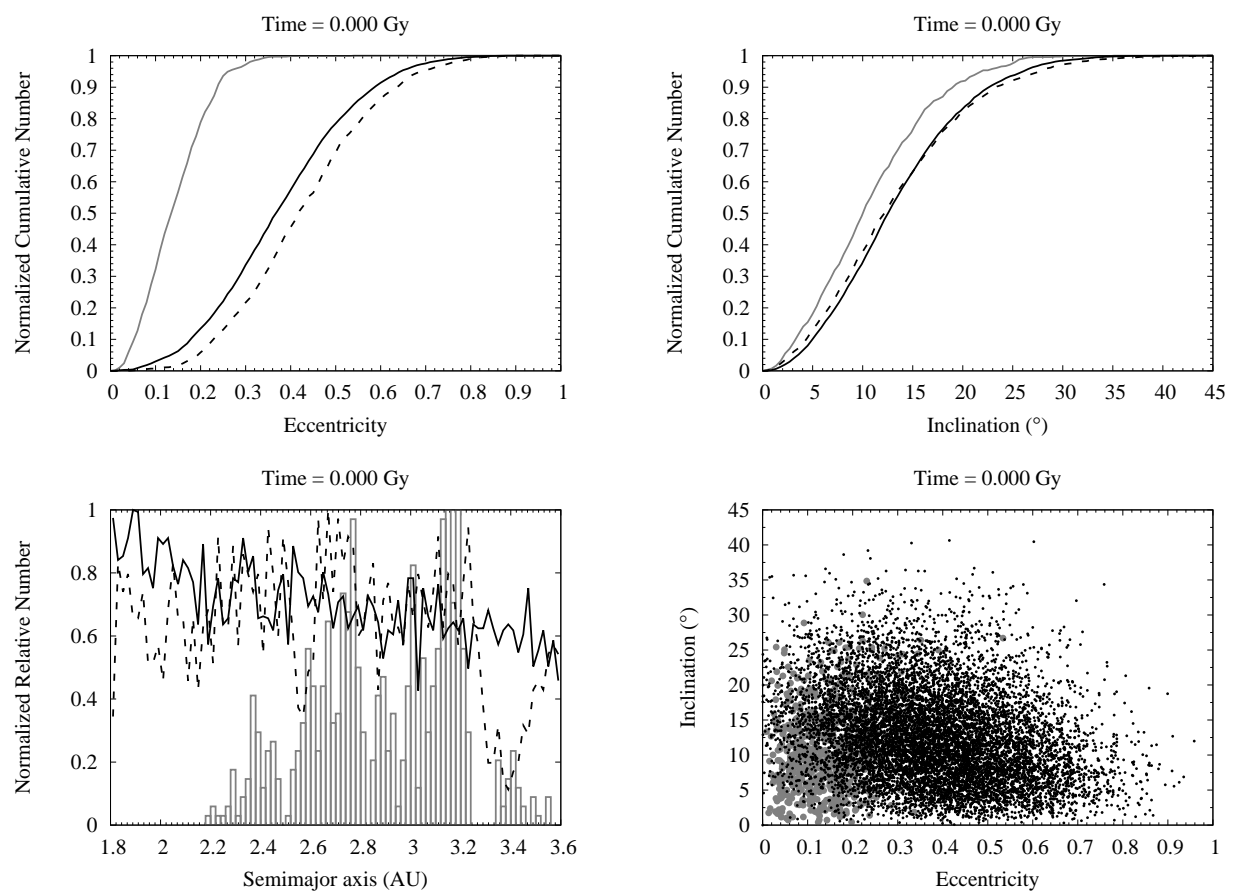

Figure 3: Normalized cumulative number of asteroids as a function of the eccentricity (top left) and inclination (top right). Normalized relative number of asteroids as a function of semimajor axis (bottom left). The width $\Delta a$ of the bins of the histogram is $0.02 \mathrm{AU}$ in semimajor axis. In all these panels the gray line refers to the real distribution, the solid and dashed black lines refer to the final distributions in the 2-planet and 4-planet Grand Tack simulations respectively. Bottom right: inclination as a function of the eccentricity. Large gray dots represent the real asteroids with $\mathrm{H}<10$ from the Minor Planet Center catalog. Small black dots represent our initial distribution generated as described in section 2 (the distribution at the end of the 2-planet Grand Tack simulation, i,e., after the dispersion of the gas nebula).

This extension of the main belt towards the terrestrial planets is precisely the E-belt advocated in Bottke et al. (2012).

Although not shown, the distribution after $700 \mathrm{My}$ of evolution is nearly indistinguishable from that shown in the right panel of Fig. 4. This demonstrates that the exact timing of the giant planet instability is not important for this study.

In order to better understand the evolution during the first $400 \mathrm{My}$, by looking in more detail at Fig. 进, we see that a very strong depletion $(\sim 71 \%)$ has occurred, approximately half in the first $100 \mathrm{My}$. This is due to the large collection of particles on clearly unstable orbits, as noted above. In fact, this happened mostly to objects with $e>0.4$, due to either collision with the terrestrial planets $(10 \%$ of the removed particles) or Jupiter $(1.5 \%)$ or ejections onto hyperbolic orbits ( $88 \%$ of the removed particles). Only $0.5 \%$ of the removed particles collided with the Sun. This number is much smaller than that for the current Near-Earth asteroids (Farinella et al., 1994; Gladman et al., 1997). This is because the giant planets are on more circular orbits, which makes the mean motion resonances much less effective in rising eccentricities than in the current

\footnotetext{
${ }^{2} \mathrm{An}$ animation of the entire evolution, from 0 to $4.5 \mathrm{~Gy}$, can be found electronically at extranet.on.br/rodney/rogerio/asteroids.mp4
} 

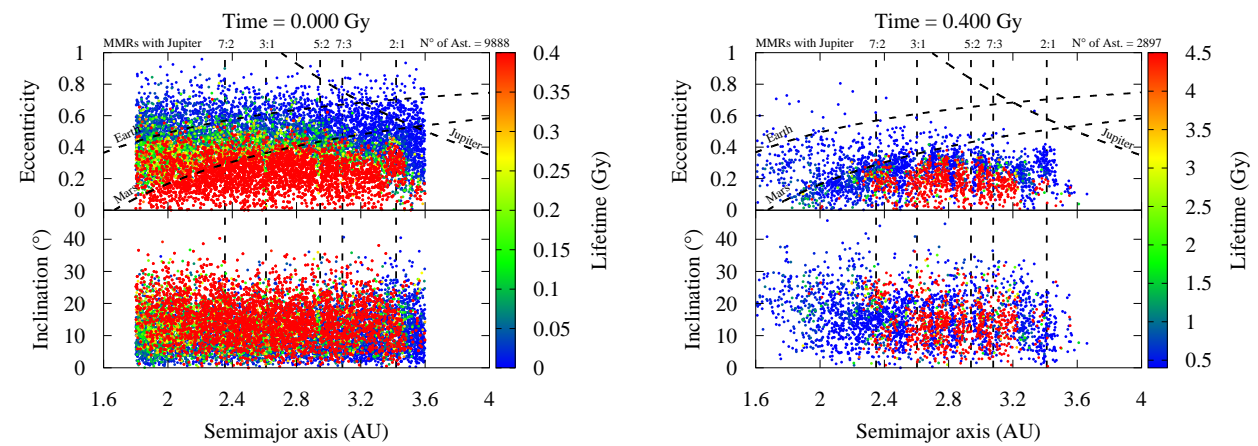

Figure 4: Left: Distribution of the particles resulting from the Grand Tack 2-planet simulation, which consitutes the initial condition for our first simulation from 0 to 400My. Right: distribution of the particles surviving at $400 \mathrm{My}$, just before the planetary instability, which constitutes the initial condition for our second simulation, from 400My to $4.5 \mathrm{~Gy}$. Particles are colored according to their lifetime in the simulation for which they represent the initial conditions. Thus, only red particles survive until the end of the simulation. Curved dashed lines in the top panels represent the boundaries for Earth-, Mars-, and Jupiter-crossing orbits (from left to right). Vertical dashed lines show the main MMRs between asteroids and Jupiter.

Solar System (mean motion resonances are stable if Jupiter is on a circular orbit and would not open gaps, Morbidelli (2002)). Also, the orbits of the giant planets are closer to each other, so the $v_{6}$ secular resonance is further out in the belt. Consequently, neither the mean motion nor the secular resonances are able to lift the particles' eccentricities to unity.

Although the distribution of particles at the end of the Grand Tack evolution covers a wide range of eccentricities, semimajor axes, and inclinations (as shown in the left panel of Fig. 4), in order to estimate the Asteroid Belt mass Walsh et al. (2011) consider only particles in the "belt region", with $q>1.9 \mathrm{AU}$ and $a<3.2 \mathrm{AU}$. In this region, our depletion ratio is $\sim 24 \%$ during the first $400 \mathrm{My}$ and $\sim 16 \%$ within the first $100 \mathrm{My}$. The Grand Tack model places $\sim 1.3 \times 10^{-3}$ $\mathrm{M}_{\oplus}$ of S-types asteroids in the "belt region", and three times as much of C-types asteroids, which implies a total mass of $\sim 5.2 \times 10^{-3} \mathrm{M}_{\oplus}$ for the post-Grand Tack main belt.

Thus, after $400 \mathrm{My}$, we still have $\sim 76 \%$ of the mass remaining in the primordial main belt, which is $\sim 3.9 \times 10^{-3} \mathrm{M}_{\oplus}$, or about 6-7 the current mass of the current Asteroid Belt $\left(\sim 6 \times 10^{-4}\right.$ $\left.\mathrm{M}_{\oplus}\right)$.

\subsection{After the planetary instability}

The second phase of the numerical simulations begin after the planetary instability. Here, as explained in section 2 , the planets are instantaneously placed onto their current orbits. With the new orbits, the secular resonances (particularly the $v_{6}$ resonance at $2.05 \mathrm{AU}$ ) appear with their full power and several mean motion resonances with Jupiter become unstable due to the larger eccentricities of Jupiter and Saturn. The lifetimes of the particles that constituted the initial conditions for this second simulation are illustrated via a color scale in the right panels of Fig. 4 .

Figure 5 shows the final orbital distribution of the particles surviving at the end of the full simulation, at $4.5 \mathrm{~Gy}$. One may note that there is a nice qualitative match between the current Asteroid Belt distribution and that produced in the simulation (compare with Fig. 2). The range of distribution in semimajor axes, eccentricities, inclinations, and the structure of the Kirkwood and secular resonant gaps look very similar in the two cases. 


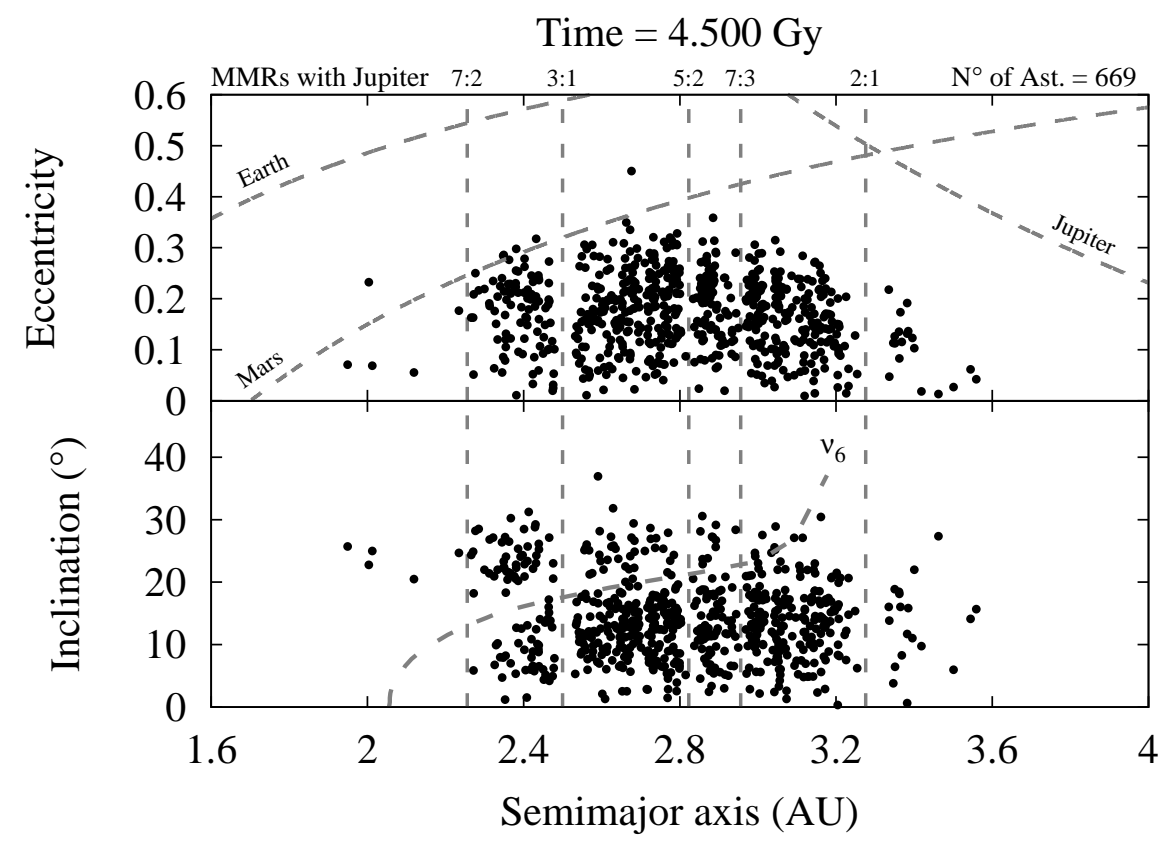

Figure 5: Configuration of the system $4.1 \mathrm{~Gy}$ after the planetary instability (i.e. at the current time). Black dots represent the asteroids the survived in the simulation. Curved dashed lines in the top panel represent the boundaries for Earth-, Mars-, and Jupiter-crossing orbits (from left to right). In the bottom panel, the curved dashed line represents the current location of the $v_{6}$ secular resonance. Vertical dashed lines show MMRs between asteroids and Jupiter.

Fig. 6 compares the final distribution of the surviving simulated particles with the current Asteroid Belt orbital distribution, in a manner similar to what we did in Fig. 3 for the initial distribution.

Fig. 6 (bottom left panel) compares the final semimajor axis distributions. The visual comparison is very satisfactory, with the exception of the zone between the 7:3 and 2:1 MMRs, which is more relatively populated by real asteroids than in our model. To be more quantitative, the fraction of the total asteroid population orbiting between major Kirkwood gaps is reported in Table 1 for both the observed and the model populations. Again, we see a fairly good agreement.

The inclination distribution (Fig. 6 top right) does not change very much (compare with Fig. 3 from the beginning to the end of our simulations, moving just slightly towards larger inclination values. Indeed low inclination asteroids are removed somewhat more frequently than high

\begin{tabular}{lllll}
\hline Kirkwood gap ratios & Zone 1 & Zone 2 & Zone 3 & Zone 4 \\
\hline Major Belt & 0.106 & 0.373 & 0.093 & 0.427 \\
Simulation & 0.147 & 0.394 & 0.141 & 0.318 \\
\hline
\end{tabular}

Table 1: Fraction of the asteroid population orbiting between major Kirkwood gaps. All fractions are caulculated dividing the number of asteroids in between two adjacent Kirkwood gaps by the total number of asteroids between the 7:2 and the 2:1 MMRs. Thus: Zone 1 represents the fraction of asteroids between the MMRs 7:2 and 3:1. Zone 2 the fraction between the 3:1 and 5:2 MMRs. Zone 3 between the 5:2 and 7:3 MMRs, and Zone 4 between the 7:3 and 2:1 MMRs. 

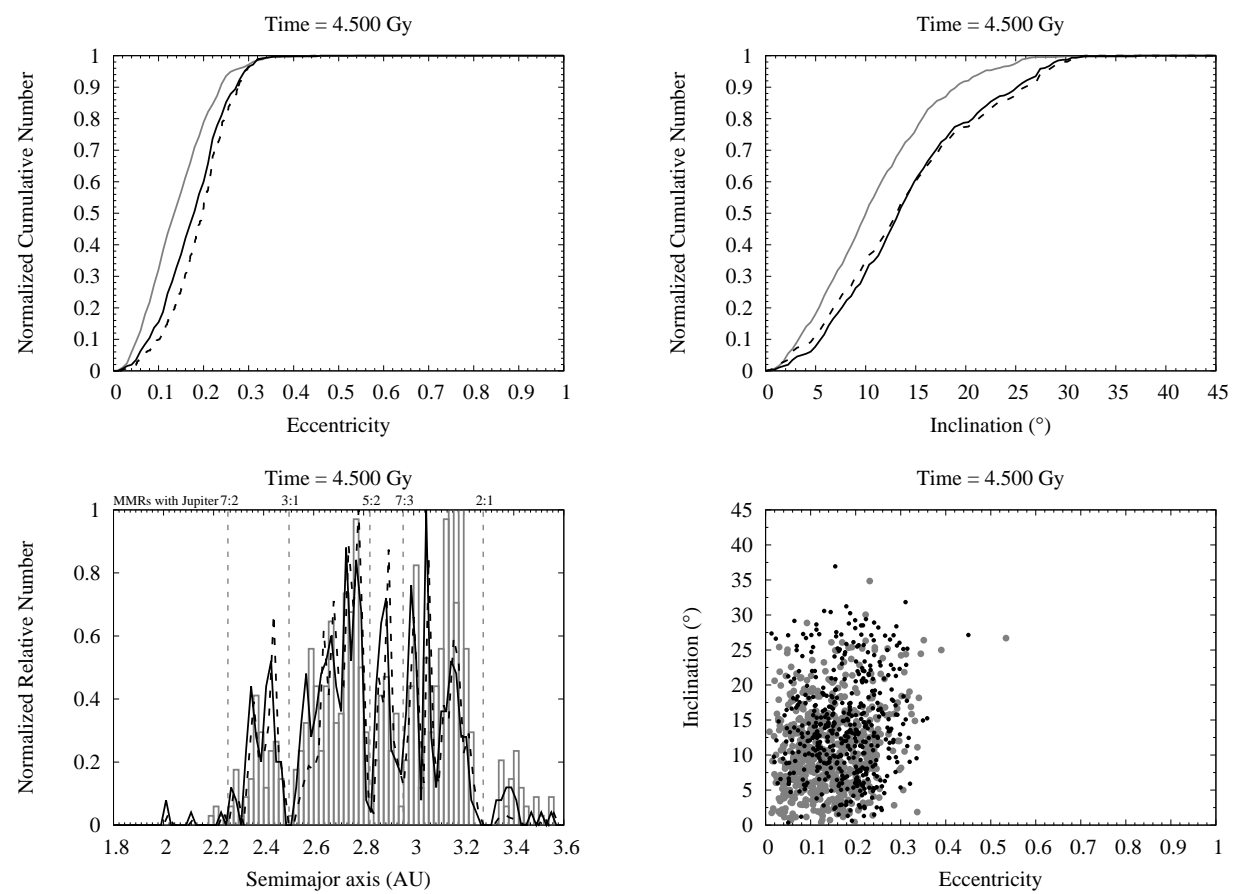

Figure 6: Normalized cumulative number of asteroids as a function of the eccentricity (top left) and inclination (top right). Normalized relative number of asteroids as a function of the semimajor axis (bottom left). The width $\Delta a$ of the bins of the histogram is $0.02 \mathrm{AU}$ for the semimajor axis. In all these panels the gray line refers to the real distribution, the solid line shows the final distribution in our simulation and the dashed line shows the distribution re-weighted according to the probability that each integrated particle exists at the end of the 4-planet Grand Tack simulation. Bottom right: inclination as a function of the eccentricity. Large gray dots represent real asteroids with $\mathrm{H}<10$ from the Minor Planet Center catalog. Small black dots represent the objects surviving after 4.5 Gy in our simulation.

inclination asteroids during the giant planet instability. Thus, our final inclination distribution is moderately more excited than the observed distribution.

This is particularly apparent in Fig. 5, in which we see (by comparison with the observed distribution in Fig. 2) that the ratio between the number of asteroids above and below the $v_{6}$ resonance in the inner belt $(a<2.5 \mathrm{AU})$ is much larger in our model $(\sim 1.2)$ than in reality $(\sim 0.09)$. However, if the initial inclination distribution (at the end of the Grand Tack) had been less excited, the final population ratio would have been much closer (for instance we obtain a population ratio of $\sim 0.1$ if we truncate the initial distribution at 20 degrees). Thus, this is clear evidence that the inclination distribution at the end of the Grand Tack is too excited. This result may suggest that the Grand Tack evolution occurred faster than simulated in Walsh et al. (2011), or that the planets had not yet reached their current masses, or there was a stronger gas-drag effect.

In the eccentricity panel (Fig. 6 top left), despite some differences that are still observed between the real and the model distributions, the match appear reasonably good, particularly if one considers how different the Grand Tack eccentricity distributions were originally (compare with Fig. 3). This shows that the Grand Tack model can be consistent with the current eccentricity distribution. 
The reason for which the final eccentricity distribution is much more in agreement with the observed distribution than the initial one is twofold. First, as explained above, large eccentricity asteroids tend to be removed because they are unstable on a timescale shorter than the Solar System lifetime. Second, when Jupiter transitions form a quasi-circular to an eccentric orbit, the asteroid's secular motion sees a value of the forced eccentricity different from before (which was almost zero). Depending on the values of the difference in longitude of perihelia between the asteroid and Jupiter when this happens, some asteroids will find themselves at the minimum of the new secular eccentricity cycle (and, possibly, by achieving a larger eccentricity they will be removed by encounters with the terrestrial planets) while others will be at the maximum of the secular eccentricity cycle. Others, of course, will be at intermediate values. By this mechanism, the mean eccentricity of some asteroids can either decrease or increase during the jump in Jupiter's eccentricity (Fig. 7). Minton and Malhotra (2011) discuss an analog process in the case of a secular resonance sweeping through an excited belt. Specifically, in our simulations we observed that $\sim 77 \%$ of the survivor asteroids kept their mean eccentricity nearly unchanged $(\Delta \bar{e}<0.05)$ when passing through the dynamical instability (Fig. 7 , bottom panel). On the other hand, within those $\sim 23 \%$ that have had their mean eccentricity changed in more than 0.05 in this phase, the ratio decrease/increase in mean eccentricity values was about half $(\sim 51 \%$ decrease and $\sim 49 \%$ increase - Fig. 7, top left and right panels, respectively). We considered the first and last $100 \mathrm{My}$ of simulation to compute and compare the mean values of eccentricities.

This reshuffling of eccentricities explains why the solid and dashed black curves in the top left panel of Fig. 6are so similar to each other. Remember (see sect. 2) that the dashed distribution is obtained from our nominal final distribution (solid curve) by weighting each integrated particle according to its probability to exist at the end of the 4-planet Grand Tack simulation. Because the distribution at the end of the 4-planet Grand Tack simulation is more skewed to large values than that in the 2-planet case (see Fig. 3), the particles in our integration with an initial low eccentricity have a weight well smaller than 1, while those with an initial large eccentricity have a weight larger than 1. Thus, if the evolution just following the Grand Tack had just preserved low-eccentricity asteroids, the final solid and dashed distribution would have been very different. Instead, the fact that a significant number of initially large- $e$ particles acquire a small eccentricity erases the initial difference in eccentricity distributions. This result is important because it shows that the evolution subsequent to the Grand Tack generates a final eccentricity distribution of asteroids that is quite insensitive of the initial one and in broad agreement with the observed one.

Concerning the mass depletion of the asteroid belt, we find that during the second phase of the simulation, from $400 \mathrm{My}$ to $4.5 \mathrm{~Gy}$, there was a depletion of $\sim 77 \%$ of the particles. This depletion is consistent with that observed in the simulations of Morbidelli et al. (2010) of the instability phase (about 50\% depletion), followed by the additional $50 \%$ depletion observed in Minton and Malhotra (2010) due to asteroids in diffusive regions, unstable on a longer timescale.

In terms of mass, we need again to consider the "belt region". In this region, the depletion from $400 \mathrm{My}$ to $4.5 \mathrm{~Gy}$ was $\sim 58 \%$. Recall that after the first $400 \mathrm{My}, \sim 76 \%$ of the particles survived in the "belt region" (representing $\sim 3.9 \times 10^{-3} \mathrm{M}_{\oplus}$ ). Thus, additionally, $\sim 2.3 \times 10^{-3} \mathrm{M}_{\oplus}$ was lost during this phase. Therefore, the final estimate for the surviving mass in the Asteroid Belt in our simulation is $\sim 1.6 \times 10^{-3} \mathrm{M}_{\oplus}$, which represents about 2-3 the current mass. Given the uncertainties in the implementations of the Grand Tack model (see the Supplementary Information in Walsh et al. (2011) for information on the wide range of test cases explored), we consider this to be a satisfactory match of the current Asteroid Belt mass.

Lastly, although there is overall consistency between our results and the current main belt, our results (bottom right panel of Fig. 6) show a lack of particles with low inclination at low eccen- 

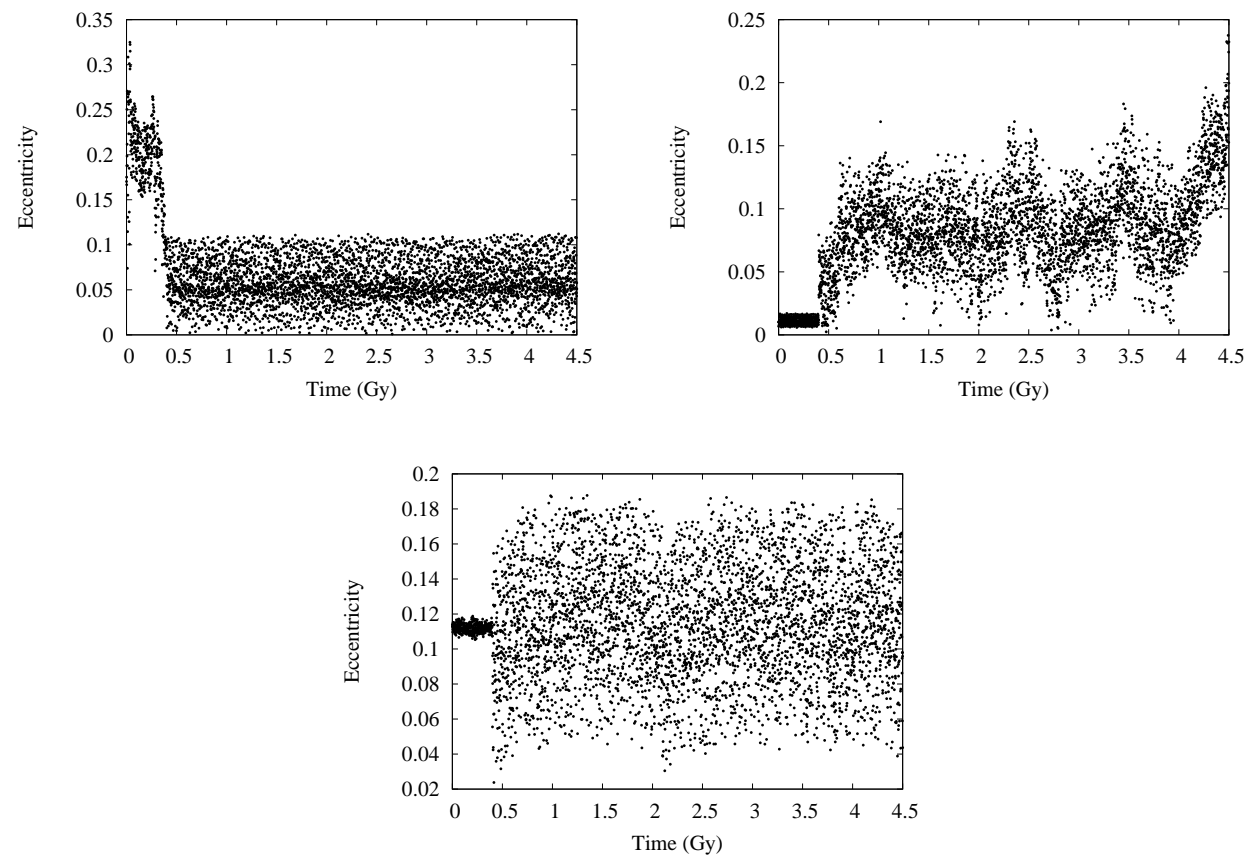

Figure 7: Evolution of the orbital eccentricity of three asteroids (test particles) during 4.5 Gy. Top left panel ilustrates a case where the eccentricity of the asteroid decreases after the LHB (at 0.4 Gy). Top right panel shows the opposite case, where the eccentricity of the asteroid increases. Bottom panel represents the majority of the cases, where only changes $<0.05$ occur in the mean eccentricity value.

tricity. In order to improve the results, either the Grand Tack simulations should have produced more particles in this region, or during the instability phase Jupiter should have had acquired a temporary orbit with a larger eccentricity than the current one, enhancing the reshuffling of the eccentricity distribution discussed above. The last option is actually likely to have taken place, because simulations of the instability (Nesvorný and Morbidelli, 2012) show that the eccentricity of Jupiter's orbit is partially damped afterwards, which implies that it had to be larger in the past. By substituting the primordial Jupiter's orbit with the current orbit, we clearly missed this phase.

Our results are complementary to those of Roig and Nesvorný (2015). In that paper the authors considered the dynamics of the giant planet instability from the most successful simulations of Nesvorný and Morbidelli (2012) but did not include the presence of the terrestrial planets. They concluded that in order to reproduce the present Main Belt, the primordial belt should have had a distribution peaked at $\sim 10^{\circ}$ in inclination and at $\sim 0.1$ in eccentricity. Here we start from the actual asteroid distribution at the end of the Grand Tack model, include the effects of terrestrial planets, but simplify the dynamical instability phase. We find that the Grand Tack inclination distribution is indeed peaked at 10 degrees and the eccentricity distribution, once reshaped mostly due to scattering by the terrestrial planets, rapidly evolves to one that peaks at about 0.1-0.2. Hence, our final distribution is in good agreement with the observed one, with a sight deficit of asteroids in orbits with low eccentricity and a slight overabundance of large inclination bodies. 


\subsection{Primordial eccentricity of Jupiter and Saturn}

Up to this point we have only considered pre-instability orbits of the giant planets as shown in Case 1 (Fig. 11 left, section 2). Here we explore the putative case of more eccentric preinstability giant planet orbits, Case 2 (Fig. 1 right, section 2), and compare with the results of Case 1 presented above.

The comparison between the results of the simulations in Case 1 and Case 2 is shown with snapshots of the evolution in Fig. 8 (with Case 1 in the left column and Case 2 in the right column) and final distribution histograms in Fig. 9 The distributions are shown both before and after the planetary instability phases (up to $1.5 \mathrm{~Gy}$ ). Case 2 results in a larger depletion. After 0.4 Gy Case 1 lost $72 \%$, and Case $284 \%$ of the primordial asteroids.

More importantly, Case 2 shows a wide gap just outside of the 3:1 MMR at 2.5 AU. This gap is not visible in the simulation of Case 1 and it does not exist in today's Asteroid Belt. Such a gap corresponds to the location of the 3:1 mean motion resonance with Jupiter, when Jupiter was on the pre-instability orbit at $a=5.4 \mathrm{AU}$. With the moderate eccentricity orbits of the giant planets considered in Case 2, the resonance is unstable and opens a gap in the asteroid distribution in $400 \mathrm{My}$. Assuming that the instability happened late, so that there is enough time to open the gap at the 3:1 MMR and that there was no interloper planet that would cause scattering of the asteroids' semimajor axes, this gap becomes fossilized and is never refilled in the subsequent, post-instability evolution. The same is true for other resonances with Jupiter (5:2 MMR at $\sim 2.9$ $\mathrm{AU}, 7: 3 \mathrm{MMR}$ at $~ 3.1 \mathrm{AU}, 2: 1 \mathrm{MMR}$ at 3.4 AU). Instead, in Case 1, where the eccentricities of the giant planets are much smaller, these resonances are far less powerful and do not open gaps. This result is consistent with that in Morbidelli et al. (2010) and constrains the eccentricities of Jupiter and Saturn characterizing their pre-instability orbits, i.e., the primordial eccentricities of Jupiter and Saturn were likely quite low (as in Case 1 simulation).

\footnotetext{
${ }^{3}$ we did not extend the simulation to 4.5 Gy because after 1.5 Gy the structure of the Asteroid Belt does not change much over time, as one may see by comparing Figs. 6 and 9 (bottom left panel), as well as by the analysis of the animation found electronically at extranet.on.br/rodney/rogerio/asteroids.mp4
} 

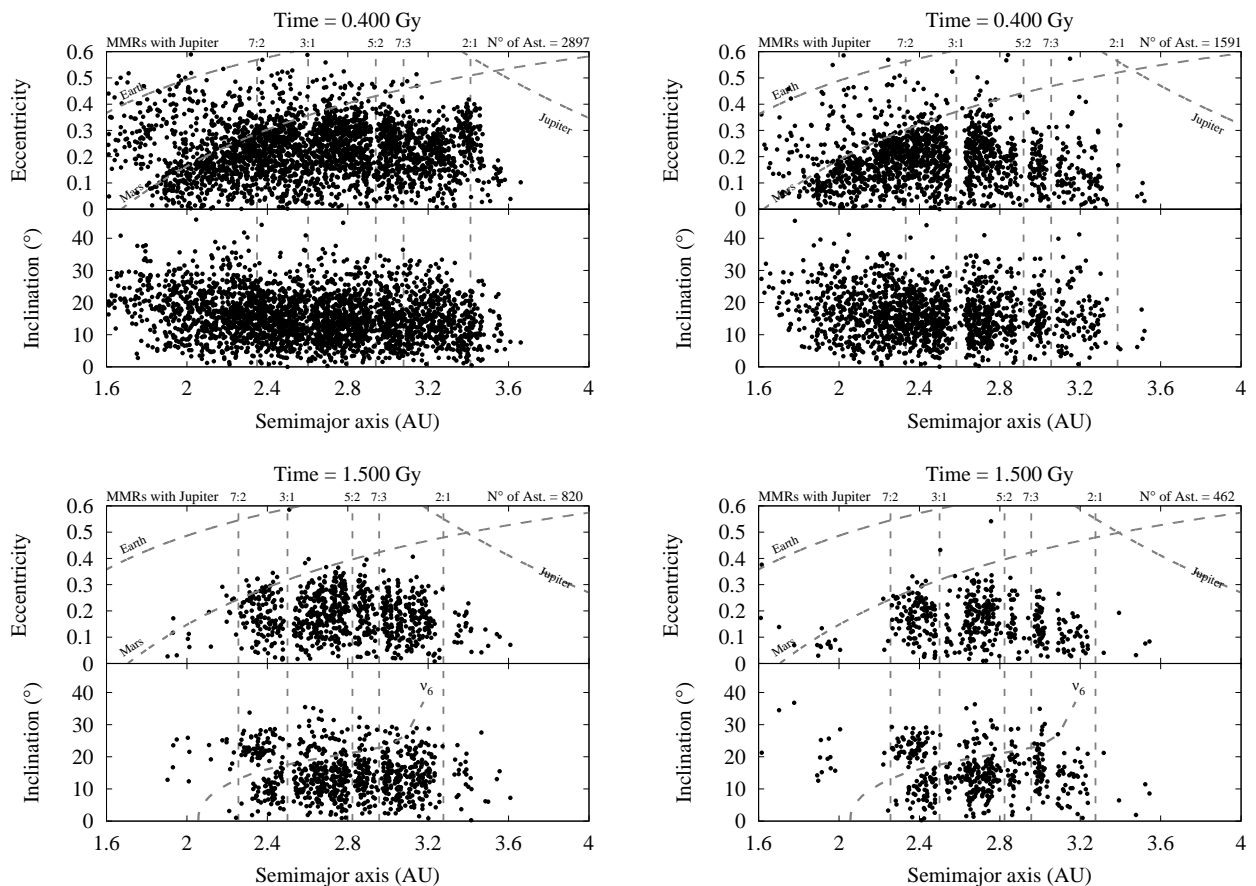

Figure 8: Snapshots of the evolution comparing the results obtained in the simulations of Case 1 (circular Jupiter and Saturn) and Case 2 (eccentric Jupiter and Saturn). Left column: Case 1. Right column: Case 2. The evolutions of Jupiter and Saturn in both cases, before the planetary instability, are shown in Fig. 1] Top: Before the planetary instability. Bottom: after the planetary instability. Curved dashed lines in the top of each panel represent the boundaries for Earth, Mars-, and Jupiter-crossing orbits (from left to right). In the bottom of the bottom panels, the curved dashed line represents the current location of the $v_{6}$ secular resonance. Vertical dashed lines show MMRs between asteroids and Jupiter.

\section{Conclusions}

In this paper we evaluated the evolution of the primordial Asteroid Belt resulting from the Grand Tack model throughout the entire age of the Solar System. We assumed that the configuration just after the end of the Grand Tack phase is the one that emerges when the gas nebula dissipates and only solid material remains in the Asteroid Belt and planetary regions. The Grand Tack model can explain the mixing of the taxonomic classes of the bodies within the asteroidal region. However, as a major drawback, it predicts a primordial Asteroid Belt that seems too excited when compared to the real Asteroid Belt.

In order to see how the subsequent evolution of the Solar System would affect the excited primordial belt, and if the Grand Tack model predictions are coherent with the current Asteroid Belt, we considered two phases of evolution:

- The first dates back to the first $400 \mathrm{My}$, before the planetary instability, but after the terrestrial planets have been fully formed in orbits more planar and circular than today (Brasser et al., 2013). Also in this phase we considered Jupiter and Saturn locked in their 3:2 MMR with Jupiter initially at $a=5.4 \mathrm{AU}$, with both planets in quasi planar and circular orbits as well. During this phase we noticed a huge depletion of the asteroid population 

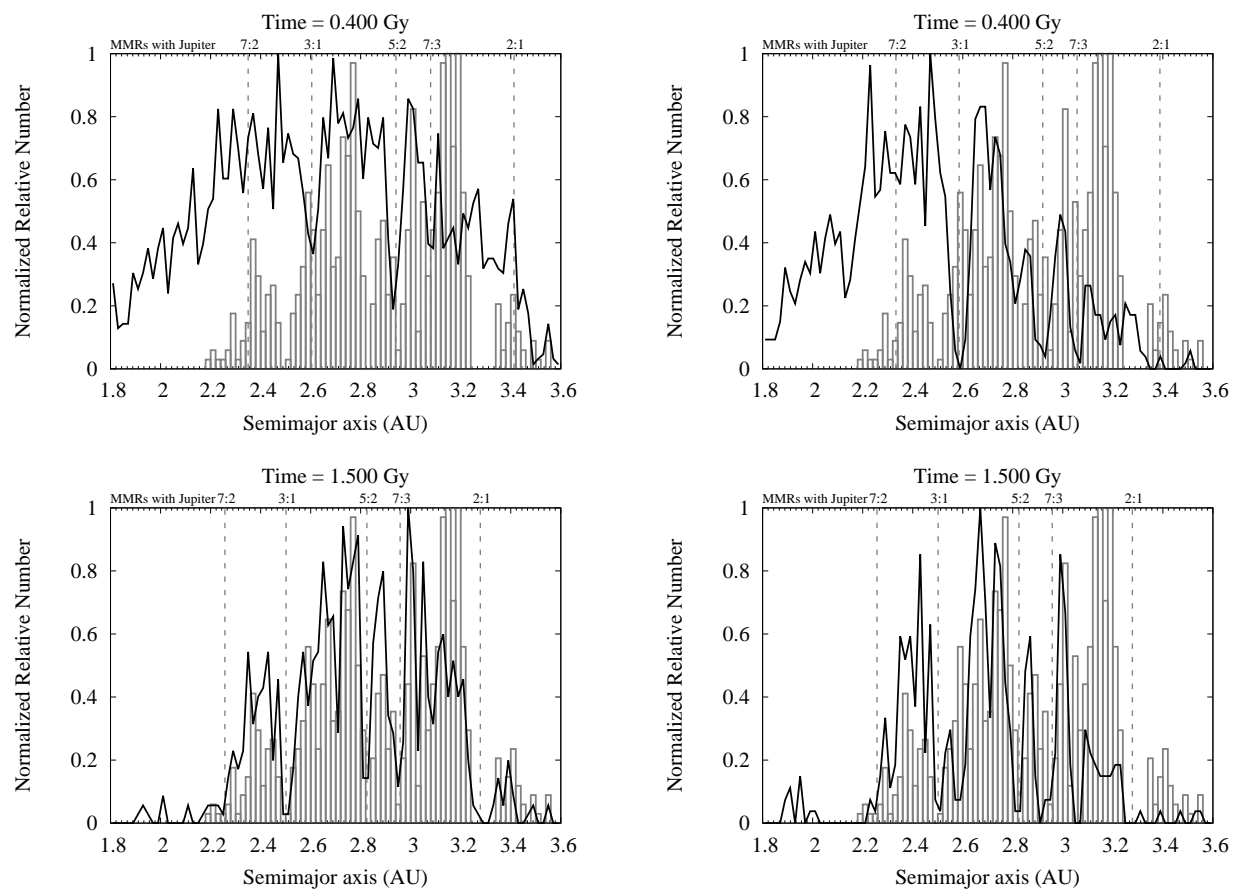

Figure 9: Comparison of the semimajor axis distributions obtained at the end of the simulations of Case 1 (circular Jupiter and Saturn, left panels) and Case 2 (eccentric Jupiter and Saturn, right panels). Top: asteroid distribution before the planetary instability. Bottom: after the planetary instability.

(about $\sim 71 \%$ ), mostly due to ejection $(\sim 88 \%)$. Most of the removed asteroids had initially $e>0.4$. Thus, in the "belt region" ( $q>1.9 \mathrm{AU}$ and $a<3.2 \mathrm{AU})$ only about $24 \%$ of the initial mass was lost, leaving $\sim 3.9 \times 10^{-3} M_{\oplus}(6-7$ the current mass of the current Asteroid Belt).

- The second phase starts just after the end of the planetary instability. We simulated the planetary instability as a discontinuous event, by instantaneously moving all planets to their current orbits. In other words, as explained in section 2 , we considered that the planetary instability happened fast enough that the actual dynamical path that the planets followed between the transition from the pre-instability orbits to the current orbits had no important effects. Thus, by using the current planetary system, the locations and strengths of all resonances match exactly the real ones, and we could simulate the response of the Asteroid Belt to the change of the planetary orbits. This phase was carried from $400 \mathrm{My}$ to $4.5 \mathrm{~Gy}$ and as a major result, it substantially changed the eccentricity distribution that, at the end, mostly resembles the current Asteroid Belt.

During the second phase, when the MMRs with Jupiter are unstable, and the $v_{6}$ destabilizes the extended part of the Asteroid Belt (E-Belt), another period of strong depletion occurs ( $\sim 77 \%$ of the bodies remaining from phase 1 were lost). Again, most of the depletion is due to ejections and concerns bodies not in the "belt region". In terms of the remaining mass, the "belt region" had an additional $\sim 58 \%$ of loss, ending with $\sim 1.6 \times 10^{-3} M_{\oplus}$ (2-3 the current mass of the cur- 
rent Asteroid Belt), which is a satisfactory match of the current Asteroid Belt's mass given the uncertainties in the implementations of the Grand Tack model.

As for the orbital structure of the final Asteroid Belt, even considering that we have a deficit of objects with low eccentricity with low inclinations in our final distribution, the results are quite good. In fact, the inclination distribution evolves slightly towards a more excited distribution and is in the end somewhat too excited relative to the observed one. We infer that the inclination distribution at the end of the Grand Tack phase should have extended only up to $\sim 20^{\circ}$, instead of $\sim 30^{\circ}$ as in Walsh et al. (2011). The semimajor axis distribution evolves from an almost uniform distribution to one quite similiar to that of the real Asteroid Belt, not only in terms of location of Kirkwood gaps but also in terms of the relative number of objects in each sub-belt region. The eccentricity distribution produced by the Grand Tack, initially skewed to large values, evolves to one similar to the observed distribution once we take into account the post-Grand Tack dynamics. Remarkably, this is the case both starting from the final distributions in the 2-planet and 4-planet Grand Tack simulations, despite these distributions being quite different from each other. The fact that Roig and Nesvorný (2015), with a very different approach, found a similar result gives support to the claim that the Grand Tack scenario is compatible with the current properties of the Asteroid Belt.

Finally, assuming that the instability happened late and that there was no interloper planet that would disperse the semimajor axes of the asteroids during the planetary instability, we show that primordial eccentric orbits for Jupiter and Saturn would have produced fossil Kirkwood gaps at misplaced positions that are not observed. Thus, our results support the idea that the orbits of the giant planets remained quasi-circular until the time of the instability.

\section{Acknowledgments}

The authors would like to thank David P. O'Brien and Nathan Kaib for the detailed review, comments, and suggestions made to improve the manuscript. R.D. acknowledges support provided by grants \# 2014/02013-5 and \#2015/18682-6, São Paulo Research Foundation (FAPESP) and CAPES. R. S. G. was supported by CNPq (grant \# 307009/2014-9). K. J. W. acknowledges support through I-SET, a NASA SSERVI program at SwRI. A. M. acknowledges support from ANR project number ANR-13\{13-BS05-0003-01 projet MOJO. D. N. was supported by NASA's Outer Planet Research program.

\section{References}

Agnor, C. B., Canup, R. M., Levison, H. F. 1999. On the Character and Consequences of Large Impacts in the Late Stage of Terrestrial Planet Formation. Icarus 142, 219-237.

Bottke, W. F., Levison, H. F., Nesvorný, D., Dones, L. 2007. Can planetesimals left over from terrestrial planet formation produce the lunar Late Heavy Bombardment?. Icarus 190, 203-223.

Bottke, W. F., Vokrouhlický, D., Minton, D., Nesvorný, D., Morbidelli, A., Brasser, R., Simonson, B., Levison, H. F. 2012. An Archaean heavy bombardment from a destabilized extension of the asteroid belt. Nature 485, 78-81.

Brasil, P. I. O., Roig, F., Nesvorný, D., Carruba, V., Aljbaae, S., Huaman, M. E. 2016. Dynamical dispersal of primordial asteroid families. Icarus 266, 142-151.

Brasser, R., Morbidelli, A., Gomes, R., Tsiganis, K., Levison, H. F. 2009. Constructing the secular architecture of the solar system II: the terrestrial planets. Astronomy and Astrophysics 507, 1053-1065.

Brasser, R., Walsh, K. J., Nesvorný, D. 2013. Constraining the primordial orbits of the terrestrial planets. Monthly Notices of the Royal Astronomical Society 433, 3417-3427.

Chambers, J. E., Wetherill, G. W. 1998. Making the Terrestrial Planets: N-Body Integrations of Planetary Embryos in Three Dimensions. Icarus 136, 304-327. 
Chambers, J. E. 1999. A hybrid symplectic integrator that permits close encounters between massive bodies. Monthly Notices of the Royal Astronomical Society 304, 793-799.

Chambers, J. E. 2001. Making More Terrestrial Planets. Icarus 152, 205-224.

Chapman, C. R., Cohen, B. A., Grinspoon, D. H. 2007. What are the real constraints on the existence and magnitude of the late heavy bombardment?. Icarus 189, 233-245.

Cumming, A., Butler, R. P., Marcy, G. W., Vogt, S. S., Wright, J. T., Fischer, D. A. 2008. The Keck Planet Search: Detectability and the Minimum Mass and Orbital Period Distribution of Extrasolar Planets. Publications of the Astronomical Society of the Pacific 120, 531-554.

D’Angelo, G., Marzari, F. 2012. Outward Migration of Jupiter and Saturn in Evolved Gaseous Disks. The Astrophysical Journal 757, 50.

DeMeo, F. E., Carry, B. 2014. Solar System evolution from compositional mapping of the asteroid belt. Nature 505, 629-634.

Farinella, P., Froeschlé, C., Froeschlé, C., Gonczi, R., Hahn, G., Morbidelli, A., Valsecchi, G. B. 1994. Asteroids falling into the Sun. Nature 371, 314-317.

Gladman, B. J., Migliorini, F., Morbidelli, A., Zappala, V., Michel, P., Cellino, A., Froeschle, C., Levison, H. F., Bailey, M., Duncan, M. 1997. Dynamical lifetimes of objects injected into asteroid belt resonances. Science 277, $197-201$.

Gomes, R., Levison, H. F., Tsiganis, K., Morbidelli, A. 2005. Origin of the cataclysmic Late Heavy Bombardment period of the terrestrial planets. Nature 435, 466-469.

Hansen, B. M. S. 2009. Formation of the Terrestrial Planets from a Narrow Annulus. The Astrophysical Journal 703 , 1131-1140.

Hartmann, W. K., Ryder, G., Dones, L., Grinspoon, D. 2000. The Time-Dependent Intense Bombardment of the Primordial Earth/Moon System. Origin of the Earth and Moon 493-512.

Jedicke, R., Larsen, J., Spahr, T. 2002. Observational Selection Effects in Asteroid Surveys. Asteroids III 71-87.

Levison, H. F., Morbidelli, A., Tsiganis, K., Nesvorný, D., Gomes, R. 2011. Late Orbital Instabilities in the Outer Planets Induced by Interaction with a Self-gravitating Planetesimal Disk. The Astronomical Journal 142, 152.

Marchi, S., and 10 colleagues 2013. High-velocity collisions from the lunar cataclysm recorded in asteroidal meteorites. Nature Geoscience 6, 303-307.

Masset, F., Snellgrove, M. 2001. Reversing type II migration: resonance trapping of a lighter giant protoplanet. Monthly Notices of the Royal Astronomical Society 320, L55-L59.

Minton, D. A., Malhotra, R. 2010. Dynamical erosion of the asteroid belt and implications for large impacts in the inner Solar System. Icarus 207, 744-757.

Minton, D. A., Malhotra, R. 2011. Secular Resonance Sweeping of the Main Asteroid Belt During Planet Migration. The Astrophysical Journal 732, 53.

Morbidelli A., 2002. Modern Celestial Mechanics: aspects of Solar System dynamics, in "Advances in Astronomy and Astrophysics", Taylor \& Francis, London.

Morbidelli, A., Tsiganis, K., Crida, A., Levison, H. F., Gomes, R. 2007. Dynamics of the Giant Planets of the Solar System in the Gaseous Protoplanetary Disk and Their Relationship to the Current Orbital Architecture. The Astronomical Journal 134, 1790-1798.

Morbidelli, A., Brasser, R., Gomes, R., Levison, H. F., Tsiganis, K. 2010. Evidence from the Asteroid Belt for a Violent Past Evolution of Jupiter's Orbit. The Astronomical Journal 140, 1391-1401.

Morbidelli, A., Marchi, S., Bottke, W. F., Kring, D. A. 2012. A sawtooth-like timeline for the first billion years of lunar bombardment. Earth and Planetary Science Letters 355, 144-151.

Morbidelli, A., Walsh, K.J., O’Brien, D.P., Minton, D.,A. Bottke, W.F., 2016. The Dynamical Evolution of the Asteroid Belt. in Asteroids IV, University Arizona Press.

Nesvorný, D. 2011. Young Solar System's Fifth Giant Planet?. The Astrophysical Journal 742, L22.

Nesvorný, D., Morbidelli, A. 2012. Statistical Study of the Early Solar System's Instability with Four, Five, and Six Giant Planets. The Astronomical Journal 144, 117.

O'Brien, D. P., Morbidelli, A., Levison, H. F. 2006. Terrestrial planet formation with strong dynamical friction. Icarus 184, 39-58.

O'Brien, D. P., Morbidelli, A., Bottke, W. F. 2007. The primordial excitation and clearing of the asteroid belt-Revisited. Icarus 191, 434-452.

Petit, J.-M., Morbidelli, A., Chambers, J. 2001. The Primordial Excitation and Clearing of the Asteroid Belt. Icarus 153, 338-347.

Petit, J.-M., Chambers, J., Franklin, F., Nagasawa, M. 2002. Primordial Excitation and Depletion of the Main Belt Asteroids III 711-723.

Petit, J.-M., Morbidelli, A., Valsecchi, G. B. 1999. Large Scattered Planetesimals and the Excitation of the Small Body Belts. Icarus 141, 367-387.

Pierens, A., Nelson, R. P. 2008. Constraints on resonant-trapping for two planets embedded in a protoplanetary disc. Astronomy and Astrophysics 482, 333-340. 
Pierens, A., Raymond, S. N. 2011. Two phase, inward-then-outward migration of Jupiter and Saturn in the gaseous solar nebula. Astronomy and Astrophysics 533, A131.

Raymond, S. N., O’Brien, D. P., Morbidelli, A., Kaib, N. A. 2009. Building the terrestrial planets: Constrained accretion in the inner Solar System. Icarus 203, 644-662.

Roig, F., Nesvorný, D. 2015. The Evolution of Asteroids in the Jumping-Jupiter Migration Model. The Astronomical Journal 150, 186

Tsiganis, K., Gomes, R., Morbidelli, A., Levison, H. F. 2005. Origin of the orbital architecture of the giant planets of the Solar System. Nature 435, 459-461.

Udry, S., Santos, N. C. 2007. Statistical Properties of Exoplanets. Annual Review of Astronomy and Astrophysics 45, 397-439.

Walsh, K. J., Morbidelli, A., Raymond, S. N., O’Brien, D. P., Mandell, A. M. 2011. A low mass for Mars from Jupiter's early gas-driven migration. Nature 475, 206-209. 